\title{
Clinical Whole-Exome Sequencing Analysis Reveals a Novel Missense COL11A1 Mutation Resulting in an 18-Week Iranian Male Aborted Fetus with Fibrochondrogenesis 1: A Case Report
}

\section{Hanifeh Mirtavoos-Mahyari}

Shahid Beheshti University of Medical Sciences School of Medicine

\section{Sanaz Ajami}

Mayo Clinic Department of Medical Genetics

Amirhosein Mehrtash

Cimorgh Medical IT Solutions

\section{Seyedeh Mahya Marashiyan}

Tehran University of Medical Sciences

\section{Farbod Bahreini}

Tarbiat Modares University Faculty of Biological Sciences

\section{Kambiz Sheikhy}

Shahid Beheshti University of Medical Sciences School of Medicine

\section{Sogol Ghanbari}

Tarbiat Modares University Faculty of Biological Sciences

Amin Ardeshirdavani ( $\square$ amin_davani@yahoo.com )

Cimorgh Medical IT solutions https://orcid.org/0000-0002-7226-7324

\section{Case report}

Keywords: Aborted Fetus, Collagen type XI a1 (COL11A1), Fibrochondrogenesis, Whole-exome sequencing (WES)

Posted Date: April 2nd, 2021

DOl: https://doi.org/10.21203/rs.3.rs-369154/v1

License: (9) (1) This work is licensed under a Creative Commons Attribution 4.0 International License. Read Full License 


\section{Abstract}

\section{Background}

Fibrochondrogenesis 1, an autosomal recessive syndrome, is an infrequent and rare disease, causing short-limbed skeletal dysplasia. This syndrome is clinically characterized and distinguished by a small nose and anteverted bares, flat midface, shortened long bones, and a protuberant abdomen. Mutations in the gene encoding the a1 chain of type XI collagen (COL11A1) are seen to be the main cause of this disease.

\section{Case Presentation}

We present an 18-week Iranian male aborted fetus with Fibrochondrogenesis 1 from consanguineous parents. Whole-exome sequencing (WES) revealed a novel missense variant from $G$ to $A$ in exon 45 of 68 in the COL11A1 gene (NM_080629.2: c.3440G>A, [p.G1147E, g.103404625]). The mutation was confirmed by Sanger sequencing and further, MutationTaster predicted this variant to be disease-causing.

\section{Conclusion}

Bioinformatic analysis suggests that this variant is highly conserved in both nucleotide and protein levels, suggesting that it has an important function in the proper role of COL11A1 protein. In-silico analysis suggests that this mutation alters the COL11A1 protein structure through a Glycine to Glutamic acid substitution. This is a novel mutation and a rare variant as this variant is not reported in gmomAD, ExAC, or 1000 genome databases.

To the best of the authors' knowledge, this is the first study to report a novel pathogenic mutation in COL11A1 in association with Fibrochondrogenesis 1. Therefore, we suggest that WES can be used as a robust method to achieve rapid diagnosis and identification of pathogenic and novel mutations in patients.

\section{Background}

Fibrochondrogenesis 1 (FBCG1; MIM 228520) is known as an autosomal congenital syndrome, which is related to short-limbed skeletal dysplasia (1). The disease is clinically distinguished by a distinctive face, protuberant eyes, flat small nose, and a small mouth accompanied by a long upper lip. Radiographically, the vertebral bodies are flat, the long bones are short and have broad, large metaphyseal ends, giving them a dumb-bell shape. Moreover, the ribs are typically short, wide, and owns metaphyseal cupping at both ends (2).

According to different researches, the disease has occurred around the world at different levels of disease, gender, and race (3). Thereby, a case of FBCG1 was diagnosed by Hunt and Vujanic in a fetus of 17 weeks, with severe micrognathia, and bifid tongue (4). Lazzaroni-Fossati and colleagues described this disorder in an infant from a consanguineous marriage (1). 
Studies have illustrated that mutations in COL11A1 are the main cause of FBCG1 incidence (2). Currently, several methods are being used for FBCG screening and diagnosis, electron microscope analysis, immunofluorescence assays, and genetic testing (2). Among these methods, genetic testing is available for the most common mutations (5). Nonetheless, rare mutations cannot be discovered using conventional methods. Clinical whole-exome sequencing (WES), as a newborn screening strategy, is an effective, successful diagnostic tool to track rare genetic alterations in a methodical and organized way for patients who have failed to receive a diagnosis despite extensive testing and follow-ups (6).

We present an analysis of the outcomes of WES that was performed in a diagnostic laboratory on an 18week Iranian male aborted fetus with FBCG1. Besides, a detailed interpretation of the identification of a novel variant in a single case with FBCG1 is described. We believe that this report can provide an insight into the phenotypic variability of this disease.

\section{Case Presentation}

The present study comprised of a single case of an Iranian aborted male fetus from a 26-years old mother. The parents were first-degree cousins. The family history for genetic or metabolic history was normal. The case was presented after 18 weeks and 2 days of gestation with absent fetal movements and multiple abnormal ultra-sonographic signs.

The disease was first clinically diagnosed by significantly small bell-shaped thorax with a protuberant abdomen, significant shortening of all limb segments despite moderately normal hands and feet, and flat midface with a small nose and anteverted nares (Figure 1a). The disease was then diagnosed with FBCG1 by Radiography. Radiographically, the ribs were typically short and wide and had metaphyseal cupping at both ends. The long bones were short and had broad metaphyseal ends, which gave them a dumb-bell shape (Figure 1b).

The genomic DNA was extracted from fetal tissue using the Omega EZNA® Tissue DNA Kit (Omega BioTek, USA) according to the manufacturer's protocols. NanoDrop spectrophotometer was used to evaluate DNA concentration $(85 \mathrm{ng} / \mathrm{ml})$. The WES was performed by Macrogen Europe (Amsterdam, The Netherlands). Target Enrichment System (Agilent, Human All Exon Kits SureSelect V7; Agilent Technologies, Inc., Santa Clara, CA, USA), followed by a paired-end high-throughput sequencing on reads of 151 bp using Illumina NovaSeq 6000 (Illumine Inc., San Diego, CA, USA). Overall, 75,309 single nucleotide polymorphisms (SNPs) and 10,068 indel variations were detected after removing low quality and out of the boundary of the capture kit in the exome analysis out of 633,000 variants (Table 1).

Obtained data from WES were first mapped to the Homo Sapiens genome reference (UCSC hg19), with mapping efficiencies of $99.9 \%$ for each paired-end read using the Burrow-Wheeler Alignment Tool (BWA 0.7.15) (7).

Variant calling of indels and SNPs was carried out by using the GenAP pipeline (Cimorgh Medical IT Solutions, Tehran, Iran) employing the Genome Analysis Toolkit (version v4.1.9.0) HaplotypeCaller 
pipeline, and the Picard tool (8). For functional annotation and genetic filtering, the GenAP automated variant annotation, classification, and prioritization were used (9). Furthermore, all the recognized recurrent mutations were confirmed using the Integrative Genomics Viewer (IGV) (version 2.8.13) (10). Following the previous step, to remove synonymous and non-exonic variants, common SNPs with minor allele frequency less than 0.02 , which were reported in the single nucleotide polymorphism database (dbSNP), the GenAP in house, the $1 \mathrm{~K}$ human genome, and the genomAD, and exAC databases were filtered out (11). Ultimately, the filtered variants were sorted based on the Combined Annotation Dependent Depletion-PHRED score (Cut-off $=15)$ and zygosity. Two homozygous variants were found in COL11A1. Only the mutation in the COL11A1, which causes FBCG1, was compatible with the clinical findings of the proband. The genomic information regarding this mutation are as follows: transcript: NM_080629.2, nucleotide change: c.3440G>A, amino acid change: p.G1147E, and chromosome position: g.103404625.

For further genetic evaluation, the rare variants with high confidence then were contemplated as diseasecausing candidates. To evaluate the pathogenicity of the novel variants, several computational algorithms including Polyphen-2, MutationTaster, and SIFT were recruited (12). Variants occurring in the well known phenotype-causing genes along with the candidate genes were selected and evaluated with priority based on known physiological, biological, and/or functional connection to the phenotype. The interpretation and elucidation of variants were conducted based on the American College of Medical Genetics and Genomics (ACMG) guidelines (13). Amongst the prioritized variants, the mutations which were predicted to be damaging and disease-causing were accounted as the most promising candidates. The results indicate that there is a missense variant from $G$ to $A$ in exon 45 of 68 in the COL11A1 gene NM_080629.2: c.3440G>A, [p.G1147E, g.103404625]), which is found to be disease-causing. This variant is highly conserved in both nucleotide and protein levels suggesting that it has an important function. This variant is not reported in gnomAD, ExAC, or 1000genome, and therefore can be considered as a rare variant.

Following the WES analysis, Sanger sequencing was performed to confirm the candidate variants found in WES as well as segregation analysis of the candidate variants within the family. The results illustrated that there is a $\mathrm{G}$ to $\mathrm{A}$ alteration at position g.103404625 c.3440. The obtained data shows that the proband carried the A/A genotype (homozygous), while the unaffected parents were found to be heterozygous for this mutation.

Finally, in-silico analyses were performed to predict the effect of this SNP on the protein structure of COL11A1. The amino acid sequence of COL11A1 in humans with NP-542196 was obtained from the NCBI server (https://www.ncbi.nlm.nih.gov). The 3D mature peptide of COL11A1 structure was built by using the iterative threading assembly refinement (I-TASSER) server, as its structure was not available on the protein data bank (14). Each model produced by the I-TASSER is given a confidence score (c-score). Higher values of the c-score indicate higher quality of the 3-D structure and higher confidence level of the predicted structure. Five models were predicted by the I-TASSER. The best-predicted model was model 1 with the highest c-score (1.28). The analysis of the predicted 3D model of proteins was validated using 
the VADAR web server (15). The Ramachandran results analysis of proteins by VADAR are as follows: Residue in phi-psi core: $70 \%$, Residue in phi-psi allowed: $24 \%$, Residue in phi-psi generous: $2 \%$, and Residue in phi-psi outside: $2 \%$. The results indicate that the selected model is applicable for mutagenesis studies. Bioinformatics predictions have reported a G1147E (G624E in predicted structure) mutation in the protein structure of COL11A1. UCSF Chimera software was then used to generate the structure and identifying the energy minimization of models. PyMOL software was recruited for visualization of structures (Figure 2a), superimposing the mutant model with the native one (Figure $2 b$ ), and evaluating the surface electrostatic potential of the native and mutated protein . Assessment of the native COL11A1 and the mutant COL11A1 displayed that the G624E mutation caused electrostatic alteration from a partial positive charge in native to the negative charge in mutant COL11A1 (Figure 2c and 2d).

\section{Discussion And Conclusions}

FBCG1 as an autosomal congenital is related to short-limbed skeletal dysplasia. According to the previous studies, the disease has two main types that are caused by mutations in the COL11A1 gene. The purpose of this study was to determine the FBCG1-causing mutation in the COL11A1 gene by performing WES on extracted genomic DNA from an Iranian aborted male fetus from consanguineous parents.

WES provided comprehensive and complete information of the sequence to screen 34 candidate genes for FBCG1. After processing raw FASTQ data, variant calling, strict variant filtering, and confirming the mutation with results obtained from Sanger sequencing, the pathogenic mutations were identified.

In this case, there was a missense variant from $\mathrm{G}$ to $\mathrm{A}$ in exon 45 of 68 in the COL11A1 gene (NM_080629.2: c.3440G>A, [p.G1147E, g.103404625]). MutationTaster predicted this variant to be disease-causing. Since this variant is highly conserved in both nucleotide and protein levels, it has an important function. As this variant is not reported in gnomAD, ExAC, or 1000genome, it can be considered a rare and novel variant. In-silico analyses have revealed that this SNP imposes a G624E mutation at the protein structure level in the predicted structure. Substitution of Glutamate, a negatively charged residue, with Glycine, a partial positive charge residue, influences the electrostatic potential, which can ultimately affect the function of the protein.

The results of bioinformatics analysis, segregation analyses, and clinical phenotype coherence have supported that the compound missense variant of COL 11A1 were the pathogenic mutations of the patient, which was first reported.

Overall, disease-causing mutations have yet to be identified for many patients with FBCG1. Many genotype-phenotype correlations remain unknown. WES provides a rapid approach to find novel mutations in FBCG1. The identification of novel mutations will improve the diagnosis and clinical treatment of FBCG1 patients.

\section{Abbreviations}


WES: Whole-exome sequencing FBCG1: Fibrochondrogenesis 1 SNP: single nucleotide polymorphism ACMG: American College of Medical Genetics and Genomics

\section{Declarations}

\section{Ethics Declaration}

The study design was approved by the ethics committee of Shahid Beheshti University of Medical Sciences. The Ethics committee approval number is IR.SBMU.NRITLD.REC.1399.197. Written informed consent was obtained from the parents of the aborted fetus.

\section{Availibility of Data and Materials}

The datasets used and/or analysed during the current study are available from the corresponding author on reasonable request.

\section{Conflict of interest}

Amin Ardeshirdavani discloses that he is an equity shareholder of Cimorgh Medical IT Solution.

Other authors declare no potential conflict of interests.

\section{Funding}

The authors did not receive financial support for any part of this article.

\section{Authors' contribution}

Conception and design of study: Hanifeh Mirtavoos-Mahyari and Sanaz Ajami

Acquisition of data: Seyedeh Mahya Marashiyan, Amirhosein Mehrtash, and Sogol Ghanbari analysis and/or interpretation of data: Kambiz Sheikhy, Farbod Bahreini, and Amin Ardeshirdavani Drafting the manuscript: Hanifeh Mirtavoos-Mahyari, Farbod Bahreini, and Sogol Ghanbari revising the manuscript critically for important intellectual content: Sanaz Ajami, Seyedeh Mahya Marashiyan, Amirhosein Mehrtash, Kambiz Sheikhy, and Amin Ardeshirdavani.

\section{Acknowledgments}

We would like to thank the parents of the aborted fetus who agreed to be part of this study. We also acknowledge the support of Shahid Beheshti University of Medical Sciences.

\section{References}


1. Lazzaroni-Fossati F, Stanescu V, Stanescu R, Serra G, Magliano P, Maroteaux P. Fibrochondrogenesis. Archives francaises de pediatrie. 1978;35(10):1096-104.

2. Tompson SW, Bacino CA, Safina NP, Bober MB, Proud VK, Funari T, et al. Fibrochondrogenesis results from mutations in the COL11A1 type XI collagen gene. The American Journal of Human Genetics. 2010;87(5):708-12.

3. Martinez-Frias M, Garcia A, Cuevas J, Rodriguez J, Urioste M. A new case of fibrochondrogenesis from Spain. Journal of medical genetics. 1996;33(5):429-31.

4. Hunt NC, Vujanić GM. Fibrochondrogenesis in a 17-week fetus: A case expanding the phenotype. American journal of medical genetics. 1998;75(3):326-9.

5. Whitley CB, Langer Jr LO, Ophoven J, Gilbert EF, Gonzalez CH, Mammel M, et al.

Fibrochondrogenesis: lethal, autosomal recessive chondrodysplasia with distinctive cartilage histopathology. American journal of medical genetics. 1984;19(2):265-75.

6. Yang Y, Muzny DM, Reid JG, Bainbridge MN, Willis A, Ward PA, et al. Clinical whole-exome sequencing for the diagnosis of mendelian disorders. New England Journal of Medicine. 2013;369(16):1502-11.

7. Li H, Durbin R. Fast and accurate short read alignment with Burrows-Wheeler transform. bioinformatics. 2009;25(14):1754-60.

8. McKenna A, Hanna M, Banks E, Sivachenko A, Cibulskis K, Kernytsky A, et al. The Genome Analysis Toolkit: a MapReduce framework for analyzing next-generation DNA sequencing data. Genome research. 2010;20(9):1297-303.

9. Wang K, Li M, Hakonarson H. ANNOVAR: functional annotation of genetic variants from highthroughput sequencing data. Nucleic acids research. 2010;38(16):e164-e.

10. Robinson JT, Thorvaldsdóttir H, Winckler W, Guttman M, Lander ES, Getz G, et al. Integrative genomics viewer. Nature biotechnology. 2011;29(1):24-6.

11. Smigielski EM, Sirotkin K, Ward M, Sherry ST. dbSNP: a database of single nucleotide polymorphisms. Nucleic acids research. 2000;28(1):352-5.

12. Adzhubei I, Jordan DM, Sunyaev SR. Predicting functional effect of human missense mutations using PolyPhen-2. Current protocols in human genetics. 2013;76(1):7.20. 1-7.. 41.

13. Green RC, Berg JS, Grody WW, Kalia SS, Korf BR, Martin CL, et al. ACMG recommendations for reporting of incidental findings in clinical exome and genome sequencing. Genetics in Medicine. 2013;15(7):565-74.

14. Roy A, Kucukural A, Zhang Y. I-TASSER: a unified platform for automated protein structure and function prediction. Nature protocols. 2010;5(4):725-38.

15. Williams CJ, Headd JJ, Moriarty NW, Prisant MG, Videau LL, Deis LN, et al. MolProbity: More and better reference data for improved all-atom structure validation. Protein Science. 2018;27(1):293-315.

\section{Tables}


Table 1. Summary of wholeexome sequencing 100X; Quality controls and Variant types

\begin{tabular}{|ll|}
\hline$\geq 10 X$ & $92 \%$ \\
\hline$\geq 30 X$ & $69 \%$ \\
\hline$\geq 50 X$ & $50 \%$ \\
\hline Mean coverage & $66.5 \mathrm{X}$ \\
\hline Total number of variants obtained & 633,000 \\
\hline Total Indel variants & 10,068 \\
\hline Exonic non-synonymous variants & 75,309 \\
\hline Frameshift & 574 \\
\hline Nonsense & 247 \\
\hline Splice site & 562 \\
\hline
\end{tabular}

\section{Figures}
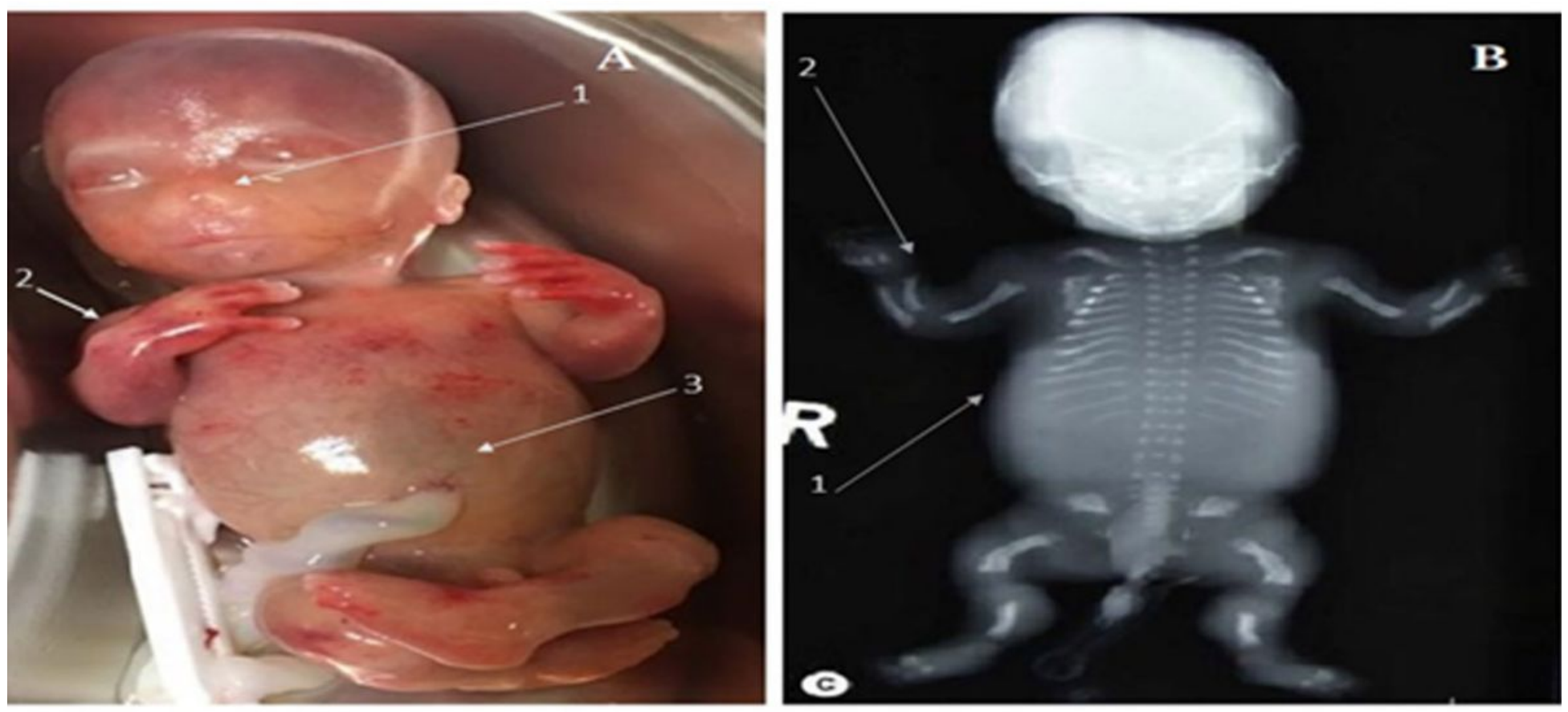

Figure 1

Clinical Photograph (A) and Radiograph (B) of Fibrochondrogenesis Case from 18-week Iranian male aborted fetus A: (1) the 18-week Iranian male aborted fetus, presenting with a flat midface, a small nose, and anteverted nares, (2) significant shortening of all limb segments but relatively normal hands and feet, and (3) small bell-shaped thorax with a protuberant abdomen. B: (1) ribs are typically short and wide and 
have metaphysical cupping at both ends, and (2) the long bones are short and have broad metaphysical ends, giving them a dumb-bell shape.
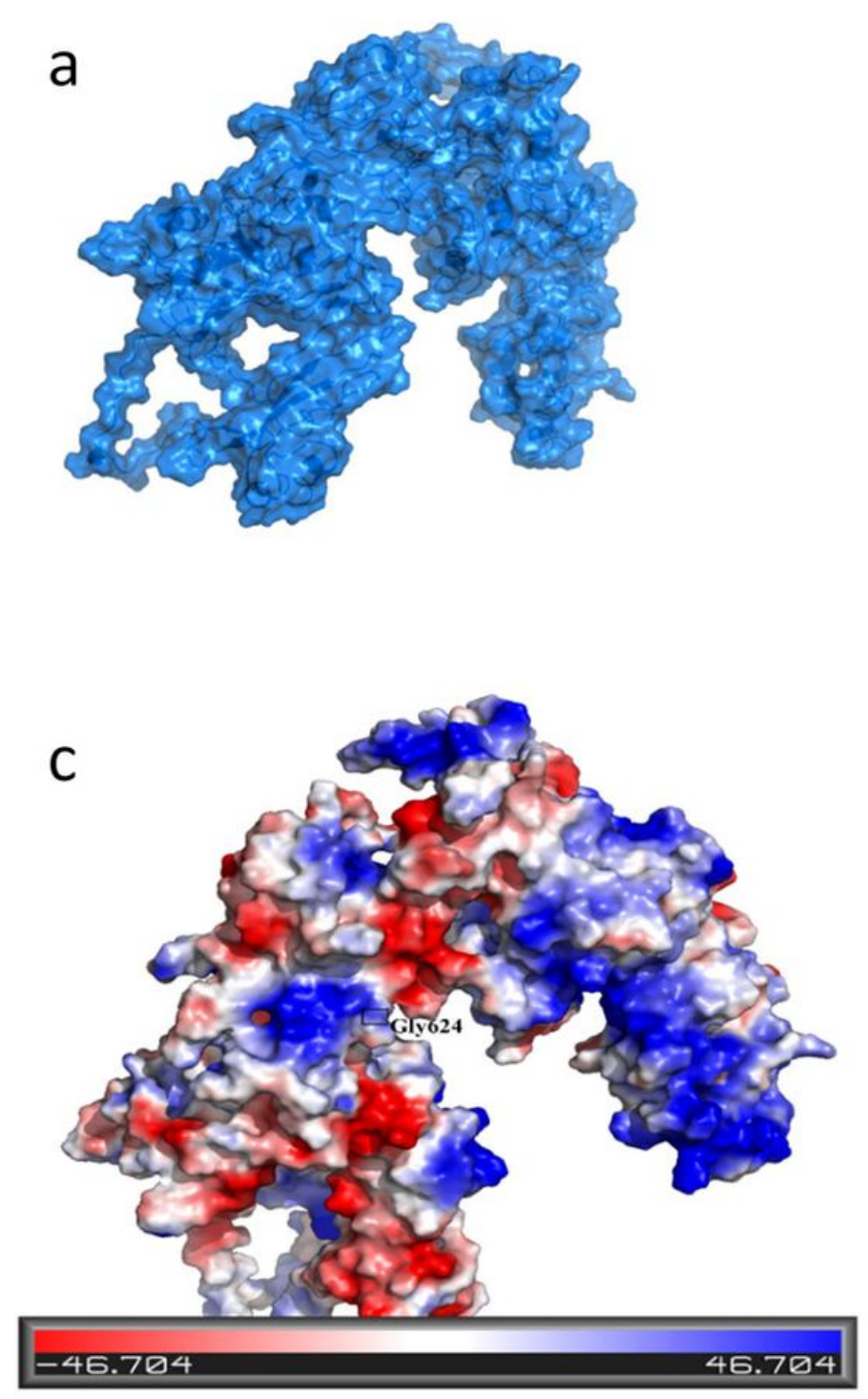
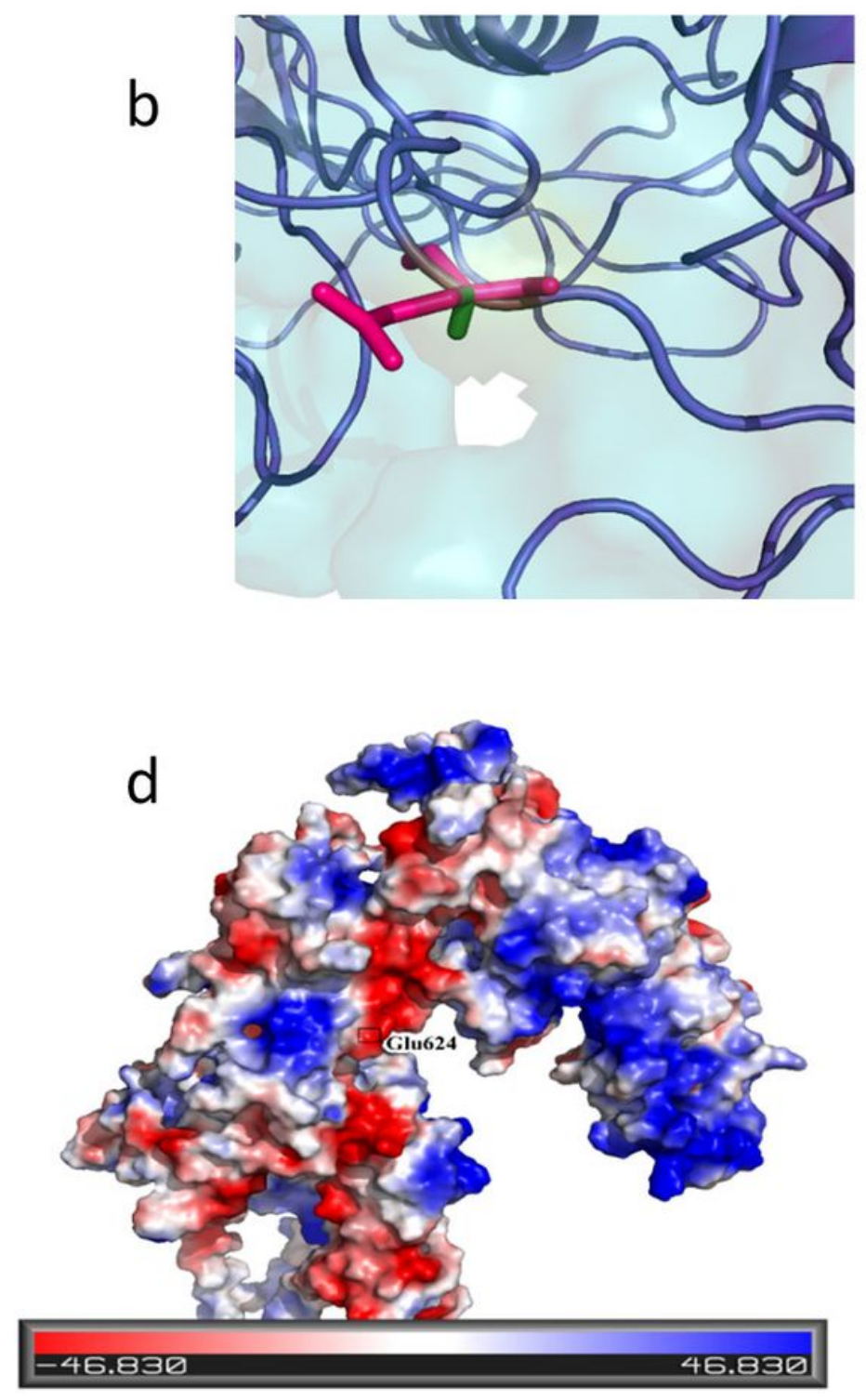

Figure 2

3D protein structure and superimposition of native and mutant structure: (a) the predicted 3D protein structure, and (b) superimposition of the native and mutant protein. Green stick represented Gly (G) in the native structure and pink represented Glu (E) in the mutant structure. (c) the surface electrostatic potential of native form and (d) the mutated form of COL11A1 were calculated by PYMOL software. Blue areas symbolize a positive charge, white a neutral charge, and the red-colored area is negatively charged.

\section{Supplementary Files}

This is a list of supplementary files associated with this preprint. Click to download.

- CAREchecklistEnglish2013.pdf 